\title{
O olhar dos gestores municipais sobre a planificação da rede temática de atenção à saúde
}

\author{
The view of municipal managers on the planning of the thematic network of health care
}

\author{
La visión de los gerentes municipales sobre la planificación de la red temática de atención \\ médica
}

Severino Azevedo de Oliveira Júnior ${ }^{1 *}$, Elem Cristina Rodrigues Chaves ${ }^{2}$, Sergio Beltrão de Andrade Lima $^{3}$; Daniela Silva Leite ${ }^{3}$, Keise Bastos Cardoso ${ }^{3}$, Ilka Lorena de Oliveira Farias Costa ${ }^{4}$, Ana Paula Oliva Reis ${ }^{4}$, Ana Tânia Lopes Sampaio ${ }^{5}$, Maria Helena Rodrigues de Mendonça ${ }^{2,3}$, Gustavo Nunes de Oliveira ${ }^{6}$.

\section{RESUMO}

Objetivo: Analisar a ótica dos gestores municipais diante das Oficinas de Planificação da Atenção Primária, realizadas na 6a Região de Saúde no Rio Grande do Norte. Métodos: Trata-se de um estudo exploratório, analítico e qualitativa realizado no período de 2011 a 2013. O percurso teórico-metodológico pautou-se na pesquisa avaliativa de quarta geração. Resultados: De forma geral, a visão da Atenção Primária é ampla, sendo reconhecida como nível de atenção essencial para desenvolver ações de promoção, proteção e recuperação, no entanto, predomina como problemática o subfinanciamento, falta de prioridade política e a desvalorização dos servidos e, referente a influência das Oficinas de Planificação e sua metodologia como instrumento organizacional da APS, foi consensual sua contribuição. Todavia, apesar dos consensos, foram apontadas contrariedades ao período político durante as oficinas, frustração dos grupos envolvidos, rotatividade dos técnicos das Oficinas e falta de apoio da gestão. Conclusão: Apesar do cenário de subfinanciamento, instabilidades política, desvalorização dos servidores e falta de apoio da gestão, foi consenso a positividade da Planificação da APS, utilizadas frente ao planejamento estratégico de ações e serviço de forma integral e regionalizada, além de ampliar conhecimentos no contexto da saúde.

Palavras-chave: Avaliação em saúde, Atenção básica, Planificação, Pesquisa qualitativa.

\section{ABSTRACT}

Objective: To analyze the perspective of municipal managers in front of the Primary Care Planning Workshops, held in the 6th Health Region of Rio Grande do Norte. Methods: This is an exploratory, analytical and qualitative study conducted from 2011 to 2013. The theoretical-methodological course was based on the fourth generation evaluative research. Results: In general, the view of Primary Care is broad, being recognized as an essential level of care to develop actions of promotion, protection and recovery, however, underfunding, lack of political priority and the devaluation of the services predominated, and, referring to the influence of the Planning Workshops and their methodology as an organizational instrument of PHC, his contribution was consensual. However, despite the consensus, setbacks were pointed out to the political period during the workshops, frustration of the groups involved, turnover of workshop technicians and lack of management support. Conclusion: Despite the scenario of underfunding, political instability, devaluation of servers and lack of management support, the positivity of PHC Planning was agreed, used in the face of strategic planning of actions and service in an integral and regionalized manner, in addition to expanding knowledge in the health context.

Keywords: Health assessment, Primary care, Planning, Qualitative research.

\section{RESUMEN}

Objetivo: Analizar la perspectiva de los gerentes municipales frente a los Talleres de Planificación de Atención Primaria, que se llevan a cabo en la 6a Región de Salud de Rio Grande do Norte. Métodos: Se trata de un estudio exploratorio, analítico y cualitativo realizado entre 2011 y 2013. El curso teórico-metodológico se basó en la investigación evaluativa de cuarta generación. Resultados: En general, la visión de la Atención Primaria es amplia, siendo reconocida como un nivel esencial de atención para desarrollar acciones de promoción, protección y recuperación, sin embargo, infrafinanciación, falta de prioridad política y la devaluación de los servicios predominante, y, refiriéndose a la influencia de los Talleres de Planificación y su metodología como instrumento organizativo de la PHC, su contribución fue consensuada. Sin embargo, a pesar del consenso, se señalaron reveses en el período político durante los talleres,

\footnotetext{
${ }^{1}$ Secretaria Municipal de Saúde de Parnamirim, Parnamirim - RN. `E-mail: juniorazevedo3719@yahoo.com.br

${ }^{2}$ Centro Universitário FIBRA, Belém - PA.

${ }^{3}$ Centro Universitário Metropolitano da Amazônia (UNIFAMAZ), Belém - PA.

${ }^{4}$ Santa Casa de Misericórdia do Pará, Belém - PA.

5 Universidade Federal do Rio Grande do Norte (UFRN), Natal - RN.

${ }^{6}$ Universidade Federal de São Carlos (UFSCar), São Carlos - SP.
} 
frustración de los grupos involucrados, rotación de los técnicos del taller y falta de apoyo de la dirección. Conclusión: A pesar del escenario de infrafinanciación, inestabilidad política, devaluación de servidores y falta de apoyo de gestión, se acordó la positividad de la planificación de la SALUD, utilizada ante la planificación estratégica de las acciones y el servicio de manera integral y regionalizada, además de ampliar el conocimiento en el contexto de la salud.

Palabras clave: Evaluación de la salud, Atención primaria, Planificación, Investigación cualitativa.

\section{INTRODUÇÃO}

A concepção ampliada de saúde como componente da seguridade social e a criação do Sistema Único de Saúde (SUS), demanda de gestores, trabalhadores e usuários com participação efetiva na construção e consensos a respeito da organização e qualificação da atenção à saúde. Diante disso, esse paradigma exige profundas transformações nas formas de planejamento e gestão dos sistemas e serviços (FAGNANI E, 2019; COSTA ILOF, et al., 2020). Neste contexto, emerge como pressuposto para elaboração de políticas públicas justas, uma programação que considere o real perfil de saúde da população e o planejamento estratégico de ações que visem responder com resolutividade os problemas de saúde no âmbito individual e coletivo (GIOVANELLA L, 2018; COSTA ILOF, et al., 2020).

O SUS apresenta-se como conjunto de ações e serviços públicos que integram uma rede regionalizada, hierarquizada, de acesso universal, visando à promoção, proteção e recuperação da saúde, organizado em três níveis de densidade tecnológica: Atenção Básica ou Atenção Primária, Média e Alta complexidade (BRASIL, 2007; GIOVANELLA L, 2018). Todavia, apesar dos avanços, é evidente a dificuldade em superar a intensa fragmentação dos serviços e qualificar a gestão na lógica da atenção integral em um sistema com predomínio de ações centradas na assistência médica e curativa organizado a partir da oferta e ao subfinanciamento, expondo-se insuficiente aos desafios sanitários e gerenciais atuais (POLATI AM, et al., 2016; MARINHO GL, 2018).

Diante as problemáticas político-institucional do SUS, emergiram discussões e iniciativas para seu aprimoramento, dentre elas, o modelo de atenção à saúde estruturado em Redes para atender de forma contínua e integral às condições agudas e crônicas da população (MENDES E, 2015; BOUSQUAT A, et al., 2017). Seguindo o desenho de redes, a Atenção Primária à Saúde (APS) constitui-se a coordenadora e porta de entrada aos cuidados e atenção à saúde que, segundo Hansen J, et al. (2015) e Bousquat A, et al. (2017), associa-se às características de serviços e ações de qualidade.

Destarte, esse processo de organização exige um planejamento estratégico, participativo e territorial, neste sentido, resgata-se a regionalização como princípio organizativo do SUS, onde a base para planificação das ações de saúde é o conhecimento do território a partir da identificação do perfil epidemiológico e sociodemográfico da população, da rede de serviços, dos recursos humanos e orçamentários existentes (BRASIL, 1988; CONASS, 2010). Assim, o Ministério da Saúde, através da Portaria de oㅜ 4279, estabelecendo diretrizes para organização das Redes de Atenção à Saúde (RAS) e busca parceria com Conselho Nacional de Secretários de Saúde (CONASS) (BRASIL, 2010).

Nesse cenário, o CONASS desenvolve as oficinas de Redes de Atenção à Saúde e propõem sua avaliação pelas equipes como um instrumento organizacional para contribuir no desenvolvimento da APS e RAS no âmbito estadual, visando a reorganização do sistema de saúde, o planejamento e gestão qualificada e a capacitação das equipes de saúde (CONASS, 2017).

O projeto de Planificação da Atenção à Saúde se destaca devido ao conjunto de ações voltadas ao corpo técnico gerencial dos estados e municípios, sendo atuantes corresponsáveis, coparticipes e cogestores das políticas de saúde e organização dos macroprocessos da APS, fortalecendo o controle social e a governança nos espaços legítimos de discussão e pactuação para uma nova lógica do Sistema de Saúde (CONASS, 2017; EVANGELISTA MJO, et al., 2019).

A origem do processo de planificação advém através da modelagem de uma "Rede de Atenção à Saúde Materno-infantil", o que foi justificado pelos problemas e dificuldades inerentes à garantia do acesso de suas 
gestantes ao parto e ao grande número de partos cirúrgicos realizados, o que tem como consequência o nascimento de bebês prematuros e um índice de mortalidade materna e infantil que preocupa e inquieta as autoridades sanitárias do estado (RIO GRANDE DO NORTE, 2010).

Através da análise documental realizada no Plano Estadual de Saúde do Rio Grande do Norte (PES/RN), pode-se comprovar que o processo de estruturação das Redes, a partir da APS, faz parte de suas diretrizes prioritárias, sendo justificadas como prerrogativas do princípio da integralidade (RN, 2012, p. 37). Dessa forma, a Secretaria de Estado da Saúde Pública do RN (SESAP/RN) adere ao processo de Planificação da Atenção Primária à Saúde, definindo um projeto piloto a ser desenvolvido na 6ª Região de Saúde do Estado (RN, 2012).

Apesar do processo das Oficinas de Planificação da Atenção Primária ter sido implementado, mesmo que parcialmente em vários estados brasileiros, é escassa produção acadêmica em torno dessas experiências, em especial no campo da avaliação e análise. Assim, o presente artigo tem por objetivo analisar a ótica dos gestores municipais diante das Oficinas de Planificação da Atenção Primária, realizadas na 6⿳⺈冂大 Região de Saúde no Rio Grande do Norte.

\section{MÉTODOS}

Trata-se de um estudo exploratório e analítico, de abordagem qualitativa desenvolvido no período de 2011 a 2013. As Oficinas ocorreram no município de Pau dos Ferros, no período de agosto de 2011 a junho de 2012, respeitando o intervalo de 45 dias entre elas. O percurso teórico-metodológico pautou-se na pesquisa avaliativa de quarta geração, passando pela construção da identificação dos grupos de interesse, constituindo círculo hermenêutico com ênfase nas reivindicações, preocupações e questões (RPQs), confronto das construções do grupo com RPQs e análise de consensos e dissensos, negociação e reciclagem (GUBA EG e LINCOLN YS, 2011).

As reivindicações são alegações favoráveis ao objeto de avaliação; preocupações são desfavoráveis e as questões são pontos de discordância, portanto, as convergências de posição avaliativa podem se expressar como reivindicações ou preocupações, e as divergências de posição como questões. Para constituição dos grupos de interesse foi considerado as posições institucionais que os sujeitos ocupavam à época da realização das Oficinas e, como critério limite para esta definição, a viabilidade de restabelecer contato com os sujeitos. Portanto, definiu-se o grupo dos 'gestores municipais' e dos 'facilitadores das oficinas' como beneficiários diretos das Oficinas".

Para estabelecer os processos de construção avaliativa conjunta, foi proposta uma sequência de atividades individuais e grupais em duas rodadas. Em função do tempo transcorrido entre 2011/2012 (realização das Oficinas) e 2013 (realização da pesquisa), no início da primeira rodada foi aplicado um questionário semi-estruturado individual de duplo objetivo: 1) estimular a memória da vivência das oficinas; 2) explicitar e verificar o posicionamento inicial dos sujeitos sobre categorias de análise mínimas de interesse do pesquisador, sendo: conceito de APS, Redes de Atenção à Saúde, ferramentas de modelagem da Rede de Atenção, satisfação com a participação nas Oficinas, território e processo de Trabalho no SUS.

Após a aplicação do questionário, foi posta técnica de grupo focal narrativo (MIRANDA L, et al., 2010), onde os pesquisadores utilizaram roteiro previamente elaborado para estimular participação em grupo e a emergência das RPQs. A atividade de grupo focal narrativo foi gravada em áudio, após consentimento livre e esclarecido ser apresentado e lido em grupo, e assinado por todos os participantes. O áudio da primeira rodada com o grupo sujeito (gestores) foi transcrito pelos pesquisadores e, a partir disso, foi construída uma narrativa do grupo, fazendo falar sua implicação, as vozes da tradição e seus interesses. A intensidade e a ênfase na qual as RPQs são explicitadas na narrativa proporcionou a construção da validação coletiva das RPQs.

O pesquisador, ao confrontar diante de si as narrativas de gestores, constrói uma narrativa pessoal que, seguindo as indicações metodológicas da Hermenêutica-Dialética e da pesquisa avaliativa de quarta geração, é um dispositivo que expõe, nos momentos de devolutiva, a visão de mundo e as interpretações da realidade, constituindo os ciclos hermenêuticos de forma participativa e democrática. 
A segunda rodada se desenvolveu inicialmente com a leitura coletiva da narrativa, no mesmo grupo da primeira rodada. A partir disso, o grupo teve oportunidade de reescrever a narrativa, retomar a discussão inicial, rever posições, reafirmar posições e complementar o texto. Encerrado este processo, o pesquisador apresenta ao grupo sua narrativa do pesquisador e se inicia mais um movimento de discussão e alteração da narrativa. Ao final da segunda rodada, o produto do grupo de interesse é uma narrativa construída e validada coletivamente, incluindo conflitos, dissensos, interesses e possibilitando processos de negociação e construção de consensos fortes em dimensão pública.

As RPQs destacadas e discutidas em cada grupo provocaram a possibilidade de ampliação da capacidade de análise e de intervenção do grupo sobre o processo vivenciado e suas consequências à 6 $6^{\underline{a}}$ Região de Saúde. Os analisadores avaliativos construídos foram aproveitados pelos grupos de interesse, servindo para disparar novos processos avaliativos em períodos posteriores, reciclando o processo avaliativo e contribuindo para ampliação e fortalecimento de uma cultura de participação e de avaliação das ações em saúde na $6^{\mathbf{a}}$ Região de Saúde. O projeto recebeu aprovação pelo Comitê Ético de Pesquisa da Liga Norte Riograndense Contra o Câncer (CAAE:19438912.8.0000.5293; Parecer no: 351.578/13).

\section{RESULTADOS E DISCUSSÃO}

Foram consideradas como categorias de análise: a percepção sobre Atenção Primária; análise da utilização das ferramentas de Planificação no processo de Modelagem da Rede Temática; Nível de satisfação em relação à metodologia das Oficinas de Planificação.

\section{A percepção sobre Atenção Primária}

De uma forma geral, considerando as respostas dos questionários aplicados, a visão em relação à Atenção Primária é bem ampla, destacando como RQPs: o subfinanciamento, falta de prioridade política nas ações, a desvalorização dos servidores que atuam nesta área e a precarização dos vínculos trabalhistas com pouco apoio para fixação de profissionais (Quadro 1).

Quadro 1 - Construção do Círculo hermenêutico-dialético em relação à percepção dos gestores e facilitadores sobre Atenção Primária.

\begin{tabular}{|c|c|c|}
\hline & Relato Grupo 1 (Facilitadores das Oficinas) & Relato Grupo 2 (Gestores Municipais) \\
\hline 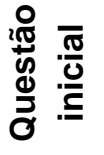 & \multicolumn{2}{|c|}{ 1. Qual sua percepção sobre Atenção Primária? } \\
\hline 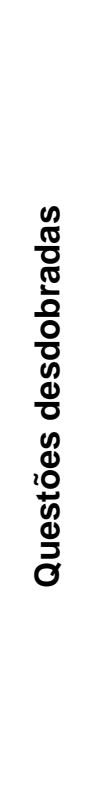 & $\begin{array}{l}\text { "É o primeiro nivel de atenção, porém onde } \\
\text { tem menos investimento"; } \\
\text { "A ordenadora de todo o sistema na teoria, } \\
\text { mas na prática ainda não exerce esse papel } \\
\text { efetivo"; } \\
\text { "Principal porta de entrada do sistema, mas } \\
\text { não garante acesso"; } \\
\text { "É o nível de atenção que define território, } \\
\text { mas a maioria dos profissionais não está } \\
\text { capacitada para realizar essa } \\
\text { territorialização"; } \\
\text { "Deve ser a ordenação do acesso, na } \\
\text { perspectiva da longitudinalidade da atenção } \\
\text { à saúde, porém há dificuldade de formação } \\
\text { de vínculo pela dificuldade de fixação de } \\
\text { profissionais". }\end{array}$ & $\begin{array}{l}\text { "Atenção primária como nível de atenção } \\
\text { essencial para desenvolver ações de } \\
\text { promoção, proteção, recuperação e e } \\
\text { reabilitação à saúde, mas ainda é pouco } \\
\text { resolutiva"; } \\
\text { "Onde se conhece de perto as diferenças } \\
\text { econômicas, sociais, culturais e o perfil } \\
\text { epidemiológico da população, mas as equipes } \\
\text { não se apoderam dessas informações para o } \\
\text { planejamento em saúde"; } \\
\text { "Onde se desenvolve o cuidado por equipes } \\
\text { multidisiplinares, no entanto, não existe ainda } \\
\text { uma estabilidade para esses profissionais"; } \\
\text { "O subfinanciamento das ações de APS e } \\
\text { pouca estruturação das redes temáticas para } \\
\text { dar suporte"; } \\
\text { "Apesar do PMAQ, a avaliação ainda é muito } \\
\text { pontual e desvinculada do desempenho de } \\
\text { qualidade". }\end{array}$ \\
\hline
\end{tabular}

Fonte: Junior SZO, et al., 2020. 
A partir da análise minuciosa das entrevistas e gravações transcritas dos grupos focais narrativos, mantendo a concepção analítica do círculo hermenêutico-dialético, foram identificadas questões desdobradas e, com isso, sintetizada uma narrativa pessoal baseada na percepção dos grupos sobre Atenção Primária (Quadro 2). Essas RQPs foram validadas na $2^{\mathrm{a}}$ rodada, com total de concordancia do grupo e acrescentada a Questão-Q em relação à viabilidade do Contrato Organizativo de Ação Pública de Saúde-COAP, sendo acordo de colaboração entre os três entes federativos recém concluido no estado. No entanto, tal Questão não foi elucidada devido, sobretudo, a irresolução da COAP quando em prática.

Quadro 2 - Síntese da análise narrativa do pesquisador em relação à percepção dos Grupos de Interesse referente à Atenção Primária.

\begin{tabular}{|l|l|}
\hline Questão: O que vocês entendem por atenção Primária? \\
\hline Análise das Narrativas & $\begin{array}{l}\text { Para eles, a Atenção Primária hoje está na gênese } \\
\text { das discussões, da mudança do modelo de } \\
\text { atenção à saúde, constituindo-se como um modo } \\
\text { de fazer em saúde que parte da identificação dos } \\
\text { problemas e necessidades da população nos seus } \\
\text { territórios. Foi consenso que sua real importância } \\
\text { consiste no planejamento estratégico de ações de } \\
\text { prevenção de doenças e agravos e promoção da } \\
\text { saúde, com coordenação do cuidado por equipes } \\
\text { multidisciplinares e ordenação do acesso, na } \\
\text { perspectiva da longitudinalidade da atenção à } \\
\text { saúde. }\end{array}$ \\
\hline
\end{tabular}

Fonte: Junior SZO, et al., 2020.

As RQPs observadas são recorrentes na literatura sendo coligadas ao modelo de gestão e planejamento adotado. Segundo Geremia DS (2020), tais problemáticas são reconhecidas como desvalorização social do SUS e atribuem-se à escassez de recursos financeiro considerando as dificuldades em atinfir um patamar razoável de investimento público.

Nesse sentido, Ribeiro LA e Scatena JH (2019) expoem a avaliação da APS como peça-chave para responder a demanda dos serviços e então impor o gerenciamento adequado das financias públicas, todavia, relatam que tal prática é limitada devido, sobretudo, à inexistencia de avaliadores qualificados para compor um campo profissional específico.

Especificamente ao financiamento do SUS, foi instituido o Piso de Atenção Básica (PAB), sendo um valor per capita a ser transferido por entes governamentais para financiar a atenção básica, destacando-se o PAB fixo, com objetivo de ampliar os serviços, reduzir as iniquidades e atribuir autonomia de planejamento (BRASIL, 1998).

Entretanto, um modelo de financiamento foi aprovada em outubro de 2019 eliminando o PAB fixo e configurando que, para os investimentos, deve ser considerado o número de usuários cadastrados e o desempenho das unidades básicas, indo em contradição com princípio de universalidade que rege o SUS e reproduzindo a seletividade na atenção à saúde (GEREMIA DS, 2020; HARZHEIM E, et al., 2020). Nesse sentido, corrobora os investimentos baseado na igualdade dos recursos às necessidades iguais, com vista na redução da desigualdade, caso contrário, as RQPs pertinentes no estudo permanecerão frequentes no futuro.

Referente a viabilidade do COAP, cujo objetivo é organizar e integrar os serviços em saúde, não foi esclarecido na pesquisa, entretanto, Teston LM, et al. (2019) expôem o olhar dos gestores municipais e pontuam a inviabilidade de pactuação ao COAP em razão às instabilidades organizativa das redes de atenção nas regiões, processo vertical da execução do contrato e ausência dos entes municipais ao cumprimeto das metas, reforçando um contexto irresolutivo e incerto às negociações interfederativa regional. 


\section{Análise da utilização das ferramentas de Planificação no processo de Modelagem da Rede Temática}

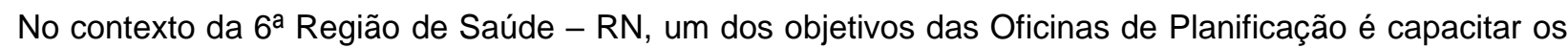
gestores e técnicos do nível local para atuar na modelagem da Rede de Atenção. Nesse sentido, foram lançadas questões relacionadas a influência das oficinas no processo de planejamento, qual a percepção dos grupos frente as ferramentas utilizada e como os conteúdos apresentados poderiam influenciar na estruturação da Rede de Atenção. A partir disso, foi observado consenso entre os grupos quanto a positividade das metodologias e ferramentas de planificação e sua contribuição ao aprimoramento profissional (Quadro 3).

Quadro 3 - Síntese da análise narrativa do pesquisador em relação à percepção dos gestores e facilitadores sobre as Oficinas de Planificação

Questão: Como o grupo analisa o processo de modelagem da Rede Temática de Atenção na 6 ${ }^{\underline{a}}$ Região de Saúde?

Análise narrativa dos facilitadores

Existiu o Consenso no sentido de que as ferramentas de Planificação no processo de modelagem disponibilizado pelas Oficinas, permitiram-Ihes se apropriar de conceitos importantes para a construção da rede, como economia de escala, parâmetros de atenção ao binômino materno-infantil e acesso. Concordam

Consensos coletivamente com a afirmação de que a metodologia desse processo é bastante empolgante, capaz de gerar interesse a todos na busca de mais conhecimento sobre os assuntos discutidos. Destacaram ainda que os produtos das oficinas foram utilizados na construção do plano da Rede Cegonha, quando conseguiram alocar os serviços com base nesses conceitos.

Análise narrativa dos gestores

A análise dos gestores quanto ao processo ocorreu de maneira bastante singular. Todos entram em consenso ao analisarem o processo de maneira positiva, citam que a visão adquirida com as mesmas ampliou o entendimento deles quanto aos serviços e até mesmo facilitou quanto a sua atuação. Sentiram-se valorizados e reconhecidos com esse processo no momento em que foram escolhidos como região piloto para implantação do projeto, gerando um sonho coletivo com o funcionamento desta rede.

Fonte: Junior SZO, et al., 2020.

Apesar da concordância de opniões, de início, os facilitadores das oficinas acrescentaram RPQs quanto a não adesão de gestores e profissionais ao exercício da teoria compreendida durante o processo e destacaram que buscam discutir e orientar os gestores com base na proposta da planificação, nas reuniões técnicas e das comissões intergestores regionais, todavia, causa não foi abraçada, sendo possível observar um cenário de não operacionalização, na prática, do processo de planificação apreendido durante as oficinas.

Diante do exposto, uma nova Questão (Q2) foi lançada ao grupo focal narrativo: As ferramentas não foram aplicadas pelos gestores por desconhecimento ou seria por falta de adesão da própria gestão ao processo? A resposta gerou uma elucidação de construção conflitante, pois veio à tona vários aspectos que corroboraram com as RPQs apresentadas, sendo observada questão a nível de poder político interferindo no cenário avaliado.

Segundo pesquisas, cerca de $94 \%$ dos participantes das Oficinas de Planificação do Rio Grande do Sul demonstraram satisfação quanto às ferramerntas utilizadas, além de expor maior contribuição nas mudanças do processo de trabalho, principalmente tratando-se da APS, Territorialização e Vigilância em Saúde e Organização da Atenção e do Processos de Trabalho (RS, 2016; DORNELES TC, 2017). 
Contudo, Megier ER (2020) evidencia que, embora os relatos das Planificações surjam como sinônimo de reorganização das redes de atenção, não é um processo inovador pois surge para expor ou relembrar profissionais de suas responsabilidades, devendo ser realizada constantemente, além de enfatizar uma falha de posicionamento da gestão, sobretudo, pela inexistência de políticas à fixação de profissionais na rede de atenção. Todavia, sua lógica possibilitou a integração entre atores relevantes na efetivação das ferramentas para gestão e ampliação do olhar quanto aos processos políticos dispostos nesse contexto.

\section{Nível de satisfação em relação à metodologia das Oficinas de Planificação}

No período da pesquisa, foram consideradas três das 11 Oficinas na 6 ${ }^{\text {a }}$ Região de Saúde Estado do RN. Nesse sentido, para identificação RPQs, foi utilizada como ponto de partida questões acerca da avaliação da modelagem, pontos fortes e fracos no trabalho das oficinas e aspectos que influenciaram o processo de Modelagem. Foi observado consenso quanto a positividade no planejamento e metodologias utilizadas nas oficinas, além de remeter a importância da participação dos gestores municipais (Quadro 4).

Quadro 4 - Síntese da análise narrativa do pesquisador sobre os consensos em relação às Oficinas de Planificação da 6ª Região de Saúde/RN.

\begin{tabular}{|c|c|}
\hline \multicolumn{2}{|c|}{ Questão 3: Como avaliam as Oficinas de Planificação? } \\
\hline Análise narrativa dos facilitadores & $\begin{array}{l}\text { Foi consenso a visão positiva em relação ao } \\
\text { preparo prévio das oficinas, no sentido de } \\
\text { solicitar informações dos municípios aos } \\
\text { participantes como base para o que seria } \\
\text { discutido, fazendo com que todos estivessem } \\
\text { instrumentalizados para trabalhar com a } \\
\text { realidade local. Outro ponto positivo citado em } \\
\text { consenso pelos facilitadores foi o planejamento } \\
\text { das oficinas, que possibilitou material para } \\
\text { estudo como apoio para esse bom planejamento. } \\
\text { Além disso, quatro facilitadores dão ênfase à } \\
\text { metodologia utilizada, relatando ser envolvente e } \\
\text { contagiante. E outros quatro concordam que } \\
\text { outro ponto forte neste trabalho foi a grande } \\
\text { participação dos municípios nas duas primeiras } \\
\text { Oficinas. }\end{array}$ \\
\hline Análise narrativa dos Gestores & $\begin{array}{l}\text { Foi consenso pelos gestores a visão de que a } \\
\text { participação deles nas Oficinas interferiu } \\
\text { diretamente no processo de modelagem da Rede } \\
\text { de Atenção na Região; a metodologia utilizada } \\
\text { como ponto forte do processo de trabalho. } \\
\text { Alguns complementam que era uma metodologia } \\
\text { dinâmica, já outros que ela permitia a } \\
\text { comparação entre teoria e realidade na qual eles } \\
\text { viviam naquele momento em seus municípios; a } \\
\text { condução do processo conta com a presença das } \\
\text { técnicas do CONASS, havendo assim uma } \\
\text { mudança com o caminhar da oficina, tendo em } \\
\text { vista que elas prenderam a atenção dos } \\
\text { participantes, assim como a vivência delas em } \\
\text { outras oficinas pelo Brasil foi bastante importante } \\
\text { para esse processo. }\end{array}$ \\
\hline
\end{tabular}

Fonte: Junior SZO, et al., 2020.

Evangelista MJO, et al. (2019) corroboram que, para uma construção eficaz das RAS, as Planificações surgem como um instrumento de gestão, principalmente a nível de territorialização, e exibe com unanimidade a importância de adotar uma cultura gestorial focada no fortalecimento da APS nessa perspectiva das redes. A metodologia de estruturação coletiva de informações e avaliação construtivista em saúde possibilita o 
conhecimento tácito, ou seja, a exposição de dimensões humanas que não se evidencia por medições e que, no entanto, têm papel decisivo no que se refere à qualidade de desempenho em relação ao serviço prestado ao usuário do sistema. Destarte, uma cogestão investigativa possibilita um controle do processo e condução das ações e serviços diante aos 3 níveis de atenção (CONASS, 2011; MEGIER ER, 2020).

Todavia, apesar dos consensos, na $1^{\text {a }}$ rodada do Grupo focal narrativo foram apontadas como RPQs: o período político durante as oficinas, pois se tratava de um ano eleitoral, portanto, responsável por parar as atividades realizadas na região, e a frustração pela decisão da Região não ser contemplada com a Rede Cegonha, mas sim como região piloto à realização das Oficinas, gerando um cenário de falta de investimentos e descrédito dos profissionais em função do abandono às demais oficinas. Ademais, foram acrescentadas à $2^{\circ}$ rodada: rotatividade dos técnicos que participavam das Oficinas; falta de apoio da gestão e as decisões da Comissão Intergestora Regional (CIR) não estavam sendo consideradas pela Gestão estadual nas deliberações e homologações da Comissão Intergestora Bipartite (CIB).

De acordo com MS (2011), a Rede Cegonha visa ampliar o acesso e a qualidade da atenção à saúde materno-infantil de forma humanizada que, segundo OMS, será investido certa 9,4 bilhões de reais para atingir toda rede de unidade básica de saúde, atribuir novas estruturas de assistência e acompanhamento e auxiliar na implantação, mobilização de gestores, monitoramento e avaliação da estratégia com meta a todo Brasil de forma gradativa (BRASIL, 2011; OMS, 2020). No entanto, no estado RN, a Rede Cegonha teve a sua operacionalização realizada pelo diagnóstico e homologação na CIB sendo escolha da $7^{a}$ região de Saúde do RN (Grande Natal) como piloto à Rede Cegonha e pela aprovação da transferência de recursos, dessa forma, tornando-se pertinentes questões de seletividade na atenção à saúde e suas consequências.

Ademais, Dornelas TC, et al. (2017), corrobora a problemática da rotatividade dos técnicos e gestores no âmbito estadual às dificuldades em redefinir e estabelecer fluxos e pactuação das RAS, portanto, não assegurando de forma efetiva às atividades estabelecidas dento da proposta de Planificação que, de acordo com autor, se estabelece como uma política de Governo, logo, dispostas a mudanças de curso a depender das gestões públicas, às instabilidade político-partidária e altercações entre governos que se sucedem no poder. Desse modo, ratifica o comprometimento gestorial à fixação e interlocução afim de estabelecer o desempenho das oficinas.

\section{CONCLUSÃO}

Apesar do cenário das RQPs referente ao subfinanciamento, instabilidades política, desvalorização dos servidores e falta de apoio da gestão, foi consenso à positividade da Planificação da APS, assim como as ferramentas e metodologia utilizadas frente ao planejamento estratégico de ações e serviço de forma integral e regionalizada, além de ampliar conhecimentos no contexto da saúde, relatando as atribuições dos profissionais, sejam da gestão ou no cuidado à saúde, como protagonismo na atenção à saúde, sendo a $6^{a}$ Região de Saúde referência para o processo de Planificação no estado do RN. Portanto, considera-se que as atividades desenvolvidas nas oficinas são de compromisso de todos e um processo contínuo de ensinoaprendizagem a ser implementado nas RAS contribuindo para seu fortalecimento.

\section{REFERÊNCIAS}

1. BOUSQUAT $A$, et al. Atenção primária à saúde e coordenação do cuidado nas regiões de saúde: perspectiva de gestores e usuários. Ciência \& Saúde Coletiva, 2017; 22(4):1141-1154.

2. BRASIL. Ministério da Saúde. Portaria no 3.925, de 13 de novembro de 1998.

3. BRASIL. Conselho Nacional de Secretários de Saúde. Assistência de Média e Alta Complexidade no SUS. Brasília: CONASS, 2007.

4. BRASIL. Ministério da Saúde. Portaria no 650, de 5 de outubro de 2011. Dispõe sobre o os Planos de Ação regional e municipal da Rede Cegonha. Diário Oficial da União, Brasília, 2011.

5. BRASIL. Ministério da Saúde. Portaria ํo 4.279, de 30 de dezembro de 2010.

6. CONSELHO NACIONAL DE SECRETÁRIOS DE SAÚDE. As oficinas para a organização das redes de atenção à saúde. Brasília: CONASS, 2010. (CONASS Documenta, n. 21).

7. CONSELHO NACIONAL DE SECRETÁRIOS DE SAÚDE. Planificação da Atenção Primária à Saúde nos Estados. Brasília: CONASS, 2011. (CONASS Documenta, n. 23). 
8. CONSELHO NACIONAL DE SECRETÁRIOS DE SAÚDE OFICINA 2 - Atenção Primária à Saúde - 18ª CRS, Rio Grande do Sul, 2017.

9. COSTA ILOF, et al. A vigilância em saúde e o planejamento nas equipes de atenção primária em saúde: revisão narrativa. Revista Eletrônica Acervo Saúde, 2020; 53:3622.

10. DORNELES TC, O residente multiprofissional na construção da Planificação da Atenção Primária em Saúde: Relato de Experiência. Santa Maria - RS, 2017.

11. EVANJELISTA MJO, et al. O Planejamento e a construção das Redes de Atenção à Saúde no DF, BrasilCiência \& Saúde Coletiva, 2019; 24(6):2115-2124.

12. FAGNANI E. Equidade e regionalização da saúde na contracorrente Cad. Saúde Pública, 2019; 35 (2): 17.

13. GEREMIA DS. Atenção Primária à Saúde em alerta: desafios da continuidade do modelo assistencial. Physis: Revista de Saúde Coletiva, Rio de Janeiro. 2020; 30(1).

14. GIOVANELLA L. Atenção básica ou atenção primária à saúde? Cad. Saúde Pública, 2018

15. GUBA EG e LINCOLN YS. Avaliação de quarta geração. Campinas: Unicamp, 2011

16. HANSEN J, et al. Living In A Country With A Strong Primary Care System Is Beneficial To People With Chronic Conditions. Health Aff (Millwood), 2015;34(9):1531-1537.

17. HARZHEIM E, et al. Novo financiamento para uma nova Atenção Primária à Saúde no Brasil. Ciência \& Saúde Coletiva, 20202;5(4):1361-1374.

18. MARINHO GL. Atenção Primária à Saúde na coordenação do cuidado e Regiões de Saúde Ciênc. Saúde Coletiva, 2018:23(12).

19. MEGIER ER. Planificação Da Atenção Primária À Saúde: Contribuições Para A 4ª Coordenadoria Regional De Saúde Do Rio Grande Do Sul. Santa Maria - RS, 2020.

20. MENDES E. A construção social da atenção primária à saúde. Brasília: CONASS; 2015.

21. MIRANDA L, et al. Dos grupos focais aos grupos focais narrativos: uma descoberta no caminho da pesquisa. In: ONOCKO CAMPOS, R. T.; et al. Tempo e Narrativa. São Paulo. WMF Martins Fontes, 2010.

22. ORGANIZAÇÃO MUNDIAL DA SAÚDE. Projeto Rede Cegonha, 2020.

23. POLATI AM, et al. Os desafios da gestão do SUS sob a ótica de gestores municipais de saúde. JMPHC. Journal of Management and Primary Health Care, 2016; 7(1):2-2.

24. RIBEIRO LA e SCATENA JH. A avaliação da atenção primária à saúde no contexto brasileiro: uma análise da produção científica entre 2007 e 2017. Saude soc., 2019; 28(2).

25. RIO GRANDE DO NORTE (Estado). Secretaria de Estado da Saúde Pública. Coordenadoria de Planejamento e Controle de Serviços de Saúde. Relatório de Gestão. Natal: SESAP, 2010.

26. RIO GRANDE DO SUL. Secretaria Estadual de Saúde. Planificação da Atenção Primária a Saúde: dados sobre avaliação, 2016.

27. TESTON LM, et al. Desafios políticos e operacionais na percepção de gestores sobre a regionalização em saúde no Acre. Saúde Debate. Rio de Janeiro, 2019; 43(121). 